\title{
Being Dignified: The Lived Experience of Elderly Chinese Residents in a Private Nursing Home in Hong Kong
}

\author{
Mimi M. H. Tiu \\ School of Nursing \& Health Studies, the Open University of Hong Kong, Hong Kong, China
}

Copyright $\bigcirc 2018$ by authors, all rights reserved. Authors agree that this article remains permanently open access under the terms of the Creative Commons Attribution License 4.0 International License

\begin{abstract}
The concept of dignity is an essential value of nursing practice and its attributes frequently include control, respect and self-esteem. In Hong Kong, elderly people have negative impressions about living in private nursing homes because they perceive these attributes of dignity will be lost. This study aims to understand and gain a deeper insight of the lived experience of being dignified for elderly Chinese residents in a private nursing home in Hong Kong. This study employed an interpretative qualitative design. A purposive sampling strategy was used to recruit 14 nursing home residents to participate in this study. The data were collected through in-depth, unstructured face-to-face interviews. Interviews were audiotaped, transcribed, and then analysed using van Manen's phenomenological method of qualitative research. Five themes that emerged from this study were: possessing inherent dignity, owning dignified-self perspectives, endeavouring to exercise dignity-conserving practices, receiving dignified care, and dwelling in a dignified home environment. Outcomes identified in this study showed the different ways dignity is conceptualised by the residents in the nursing home. The results enhance our understanding of dignity among residents in private nursing homes and can contribute to the development of strategies for providing dignified care in future.
\end{abstract}

Keywords Dignity, Elderly Chinese Residents, Nursing Home, Phenomenology

\section{Introduction}

The concept of dignity is an essential value of nursing practice. As treating someone with dignity may impact positively upon treatment and social outcomes for the elderly, promoting dignity proves to be a cost-effective measure in raising both the quality of life and quality of care for this sector of society (Tadd et al. 2002) [28]. This study aims to explore the lived experience of being dignified for elderly Chinese residents in a private nursing home, and also aims to explore the situated possibilities, caring activities, and practices that constitute - and are constitutive of - the elderly residents' perspective on maintaining dignity in the nursing home. Understanding the lived experience of elderly Chinese could contribute to develop dignified strategies in nursing homes. Thus, achieving the ultimate goal in caring for elderly residents in nursing homes - providing them with dignified care would enable them to live in dignity.

\section{The Concept of Dignity}

Dignity is described as a vague, ambiguous, highly abstract concept that is impossible to measure (Chinn \& Kramer 1999; Fenton \& Mitchell 2002; Griffin-Heslin 2005; Haddock 1996; Shotton \& Seedhouse 1998) [2, 6, 24]. It is commonly associated with self-esteem, worth, control, self-respect, respect by others, capability, and autonomy (Fenton \& Mitchell 2002; Griffin-Heslin 2005; Haddock 1996; Jacelon et al. 2004; Mairis 1994; Matiti \& Trorey 2004; Nordenfelt \& Edgar 2005; Sulmasy 2007). [4-6, 8, 15, 16, 18, 27] A number of scholars have interpreted the concept differently. For example, Pullman (1999) [21] distinguished between 'personal' and 'basic' dignity. Nordenfelf (2004) [17] distinguished between 'the dignity of merit' and 'the property of being human'. Sulmasy (2007) [27] distinguished between 'attributed' and 'intrinsic' dignity. While they may use different language to speak about how these two interrelated conceptions contribute to the overall meaning of human dignity, these concept can be categorised into two accounts, i.e. the particularist and universalist accounts. Particularist accounts refer to a particular notion of dignity in which individually or socially-valued properties or qualities discernable through empirical sensibilities describe the 
features or value of those capacities that contribute to human meaning and worthiness. Universalist accounts of dignity refer to a basic moral attitude or moral platform that gives credence to the belief that human beings enjoy an inalienable and universal inherent worth that forms the basis of the relational demands of inherent human dignity (Pullman 2001) [22].

\section{The Local Context}

With an ageing population and limited living space, ageing in place (at home) for those requiring physical assistance seems to be impossible for the majority of the elderly in Hong Kong. Thus, residential services for the elderly are important. The number of beds in residential care facilities in September 2017 was 74,245, of which 41,929 beds (56\%) were provided by private nursing homes (Social Welfare Department 2018) [25]. Private nursing homes play a significant role in providing care for the elderly in Hong Kong. With their limited resources, however, their quality varies greatly, and they are not comparable to the subsidised residential care homes. The mission of elderly services is to enable elderly people to live in dignity (Social Welfare Department 2018) [25]. To the general public, the image of private nursing homes is negative, and whether the residents in private nursing homes are living with dignity is questionable.

\section{Methods}

A hermeneutic phenomenological approach was used in this study because it was the best or only way to understand phenomena deeply and in detail (Richards \& Morse 2007) [23]. This method allows for an increase in the awareness of humans and their ways of being-in-the-world (Speziale \& Carpenter, 2007) [26]. This study was guided by van Manen's work because this methodology is open to innovation. According to Speziale \& Carpenter (2007) [26], there are four major procedural steps in using this hermeneutic approach:

The first step is to turn to the nature of lived experience by orienting to the phenomenon, formulating the phenomenological question, and explicating assumptions and pre-understanding. In this process, there is commitment to questioning the phenomenon of the study thoroughly and giving it considerate thought in order to make sense of certain aspects of human existence (Peyrovi et al. 2005) [19]. To do this phenomenological research is to question 'being dignified' phenomenologically, and to address the question of what 'being dignified' is 'really' like - or means - to the elderly residents in nursing homes.

The second step is to investigate the experience as one lives with it. This phase of this research involved collecting information about the phenomenon of being dignified which included descriptions of participant's experience with the phenomenon.

\section{Setting}

A private 300-bed nursing home located in the New Territories of Hong Kong was chosen for the study.

\section{Participants}

A purposive sampling method was used to conduct this study. The researcher recruited participants who will be most representative or informative (Polit \& Beck 2006) [20]. The inclusion criteria of participants included residents who were: i) at least 65 years old; ii) had been living in this nursing home for two years or more; iii) were willing and able to talk about their experience, and iv) able to speak in Cantonese were considered as potential participants. A total of 14 elderly residents participated in this study.

\section{Data Collection Procedure}

Data were collected from in-depth individual interviews. All interviews were audiotaped. The open-ended, unstructured interviews were conducted in a single room in the nursing home. During the interview, a broad question, 'What is your experience of being dignified?' was asked. The researcher also advised participants to describe their experience as fully as possible without making suggestions for responses. Participant observation was also used as a method to collect data in this study. All the observations obtained were recorded in the researcher's field notes. Data collection continued until saturation of data was reached.

\section{Ethical Considerations}

Prior to the conversations, an information sheet and consent was obtained from the participants. The researcher reassured the participants that their participation in the inquiry was voluntary, and they were informed of their right to refuse to participate or discontinue participation at any time throughout the duration of the study without any adverse consequences. The researcher assured that their anonymity would be maintained by assigning a code name (alphabet). The information would be kept confidential and could only be accessed by the researcher and relevant others. Any documents related to this study would be kept in a locked area and would be destroyed after publication of the data.

\section{Data Analysis}

The third step was to engage in phenomenological reflection, which involved conducting thematic analysis, uncovering thematic aspects in life-world descriptions, isolating thematic statements, and interpreting through conversation. After the interviews were transcribed, the researcher spent time reading the lived-experience descriptions in a meditative way seeking to uncover or 
isolate thematic aspects of the phenomenon. The researcher used the selective approach to derive the themes. After the themes were isolated, the researcher reflectively transformed the thematic statements into more phenomenologically-sensitive paragraphs. This reflection research process was guided by the lifeworld existentials of lived space (spatiality), lived body (corporeality), lived time (temporality), and lived human relation (relationality) (van Manen 1990) [31]. The researcher used these four lifeworld existentials to guide the theming process.

\section{Hermeneutic Writing}

The last step was to engage in phenomenological writing, which included writing and rewriting. This phase in this research process was to create a phenomenological text which included describing the lived human experience through writing; the ultimate aim of which is to move the reader to a deeper or more enlightened recognition of the phenomenon. In order to do justice to the fullness and ambiguity of the experience of being dignified for elderly residents in the nursing home, the researcher had to write, rewrite and re-think, reflect and recognise. The researcher engaged in conversational relationships with the text as well as with the participants, and was attentive to what was said and what was unsaid. In other words, the researcher was sensitively attentive to the silence around the words (van Manen 1990) [31] in attempting to disclose the deep meaning of the experience. From this complex process, the phenomenological text was created.

\section{Rigour of the Study}

To ensure the rigour of the research process, the data and process were evaluated using the criteria of credibility, dependability, transferability, and confirmability for quality research (Lincoln \& Guba 1985) [12]. To ensure credibility, prolonged engagement at the site of inquiry, persistent observation, peer debriefing, and member checks were performed. Dependability was guaranteed when credibility was assured (Peyrovi et al. 2005) [19]. Transferability was accomplished by identifying broad criteria for sample selection and purposive sampling, and providing the reader with rich in-depth descriptions that portrayed the meaning of the residents' lived experiences. Audit trail was performed to ensure the confirmability.

\section{Findings}

The lived experience of being dignified by the participants living in the nursing home revealed five major themes. They included: possessing inherent dignity, owning dignified-self perspectives, endeavouring to exercise dignity-conserving practices, receiving dignified care, and dwelling in a dignified home environment.

\section{Possessing Inherent Dignity}

Inherent dignity is the dignity that the participants perceived that one is born with. Many participants expressed that one nature or form of dignity is that it is innate. One is being dignified because he or she is a "human being", for example, Mr. C commented that:

Those who are paralysed, physically impaired, or even become unconscious have dignity. Therefore, we should treat them well and they need to be treated as human beings... as if they are normal people.

Mr. C perceived dignity as a 'something' that everyone in the nursing home should have. However, there are different levels of dignity, he said:

The ones who can walk have their dignity; so do the ones who cannot. The ones who can walk usually have a higher sense of dignity than those who cannot. The rich ones have their dignity; so do the poor. The richer one is, the higher the sense of dignity one can obtain. However, the one who has no money still has dignity...so we cannot overlook the one who has no money or those who are paralysed because they have dignity too.

As described, it was revealed that one nature of dignity is innate. Merely existing as a human being, regardless of what one has, deserves to be accorded dignity by others.

\section{Owning Dignified-self Perspectives}

This theme explicates what constitutes a dignified person in the nursing home from the 'self' perspective. Not being a burden to others and dying with dignity were the major characteristics which contributed to the residents' sense of dignity. As Ms. A stated:

I have heart failure. I don't want my family worrying about me. They need to work. Living in a nursing home can help to relieve their stress. It is beneficial for them and for me. If they do not need to worry about me, they can focus more on their jobs...it is important for me not to create problems for them.

By living in the nursing home, they have indeed enhanced their dignity as they will not, to some extent, be a burden for others. Besides not being a burden, Ms. A also related dignity with good death:

Having a good death means dying without any pain; the best way of dying is to die while I am sleeping, I will die without knowing I am dying, dying naturally and peacefully...the process should be quick...that is dignity... Well, I guess these are dream words, because nobody, nobody, can control how one dies...this part, I really have no control over...but I think I can choose not to have any life-sustaining treatment, right? It would be terrible seeing myself attached to all the machines, "tubes" or "lines". 
Residents realise there are certain things that are not under their control, such as life and death. Thus they do not attempt to control the way they die; but they definitely would not want the unnecessary prolongation of their lives. The residents perceived that by not being burdens to others and able to die without unnecessary prolongation, their dignity could be maintained.

\section{Endeavouring to Exercise Dignity-conserving Practices}

Dignity-conserving practices are the strategies the residents use to support their sense of being dignified in the nursing home. The sub-themes that emerged included having perseverance in setting and achieving goals, and making a contribution.

Having perseverance in setting and achieving a goal is a way for residents to bolster their sense of dignity. Once they had put in the effort and were able to accomplish the goal, dignity was the outcome. When Mr. D was discharged from hospital, he was unable to walk. He promised himself that he must regain the ability to walk. He said:

If I did not exercise or try to walk, I would never be able to walk again for the rest of my life. I encouraged myself that I could do it. After 6 months of practice, I was able to walk with the assistance of two of my sons. When I needed to go out, one son had to hold my left arm and the other son had to hold my right arm; I kept practicing every day. After one year, I was able to walk without any help.

For him, the process was difficult but he did not want to give up on his ability to walk. His goal was to walk without walking aids. He succeeded and expressed dignity. This example demonstrated having perseverance to overcome a difficulty and to achieve the goal is one of the strategies used to maintain dignity.

Making contributions to the nursing home is a practice for some residents to maintain their dignity. Ms. E helps other residents by reading the news to them, talking with them, and actively asking them whether any help is required. She said:

It seems I am giving them help, but indeed they are helping me. Helping me to develop my self-worth and making me feel less bored than I should be...I feel good and dignified if I can make them happy...I guess that is important to support my sense of dignity.

Many participants discussed feeling worthless as they thought they had nothing to contribute to society. It would be important to make them feel they could contribute to the nursing home; to make them feel worthy and dignified.

\section{Receiving Dignified Care}

Care was an important aspect that many of the participants discussed. It was revealed that dignity is obtained through their being embraced by the filial piety (xiao) (Teon, 2016) [29] of their children and being cared for by non-paternalistic nursing staff.

Many participants became very excited when they talked about the visit of their children, just like a new parent shared their excitement with others concerning their new baby. Ms. J, for example, did not like living in the nursing home. However, when she talked about her son's visit on Saturdays and Sundays, she started to smile and said:

He brings me expensive cakes every week... I told him not to buy them - they are too expensive but he did not listen. He said that it is important to find the food that I like. The cakes are so good!

Besides the children, the nursing staff are able to affect the residents' sense of dignity. If the staff believe that they have power over the residents, or they are domineering, they cannot develop a good relationship with residents and thus cannot deliver dignified care. The residents disliked staff that acted like their parents. For example, if the staff said, "I told you not to eat too much, your blood glucose is above the normal range. That is your fault!" or "Don't walk by yourself, you'll fall", the residents' sense of dignity will be intruded upon.

\section{Dwelling in a Dignified Home Environment}

Participants spoke about how their environment contributed to the sense of dignity. In this aspect, the meaning of dignity includes having privacy boundaries, and having appropriate devices that enhanced independence.

Some residents preferred to pay more for a single room to 'buy' privacy. Ms. B is an example. She refused to go to the assigned subsidised nursing home, for which she had been waiting five years, because there was no single room available. She preferred to pay the single room rate in this private nursing home, which is four times higher than the room rate in the subsidised nursing home, to maintain her privacy. She said:

I'll be more dignified if I can have a single room, which is a real area that really belongs to me. I don't mind to pay more for that.

The availability of devices that maintain the independence of the residents, such as an elevator, contributed to their sense of dignity. Without these devices, the residents feel indignity. The first day that Mr. M came to the nursing home was unforgettable. He was being discharged from hospital and sent to the nursing home by non-emergency ambulance. There were three other elderly men in the same ambulance who were also being sent back to nursing homes. He said:

When the first man was dropped off at the first nursing home, I thought, this building was almost as old as we are; luckily, this wasn't the one I'm going to. Then the second one arrived at his nursing home, I could never 
forget this scene. The outlook of the building was newer than the first one. It consisted of three floors with no elevator. Two staff without a wheelchair came down to pick up the second man. They picked him up in a very special way: one lady held the upper part of his body and the other held his legs. The man was covered by a white bed sheet from the hospital. It seemed they were lifting a corpse up to his room. I thought he had no dignity at all and that really frightened me. I felt sick and wanted to tell the driver I am not going to the nursing home. Fortunately, this one was different...this one had elevator and wheelchair stand by...

\section{Discussion}

Based on the above findings, a framework on being dignified in nursing home was developed (Figure1). In the framework, it was revealed that the lived experience of Chinese residents in a private nursing home included their perception of dignity and their strategies to conserve their dignity. Inherent dignity, together with dignified-self perspectives, is the perception of dignity. The residents themselves, the family members and nursing staff, and the nursing home environment can provide strategies to conserve dignity. These strategies and the dignified-self perspectives are interrelated.

\section{Endeavouring \\ to exercise \\ dignity- \\ conserving \\ practices}

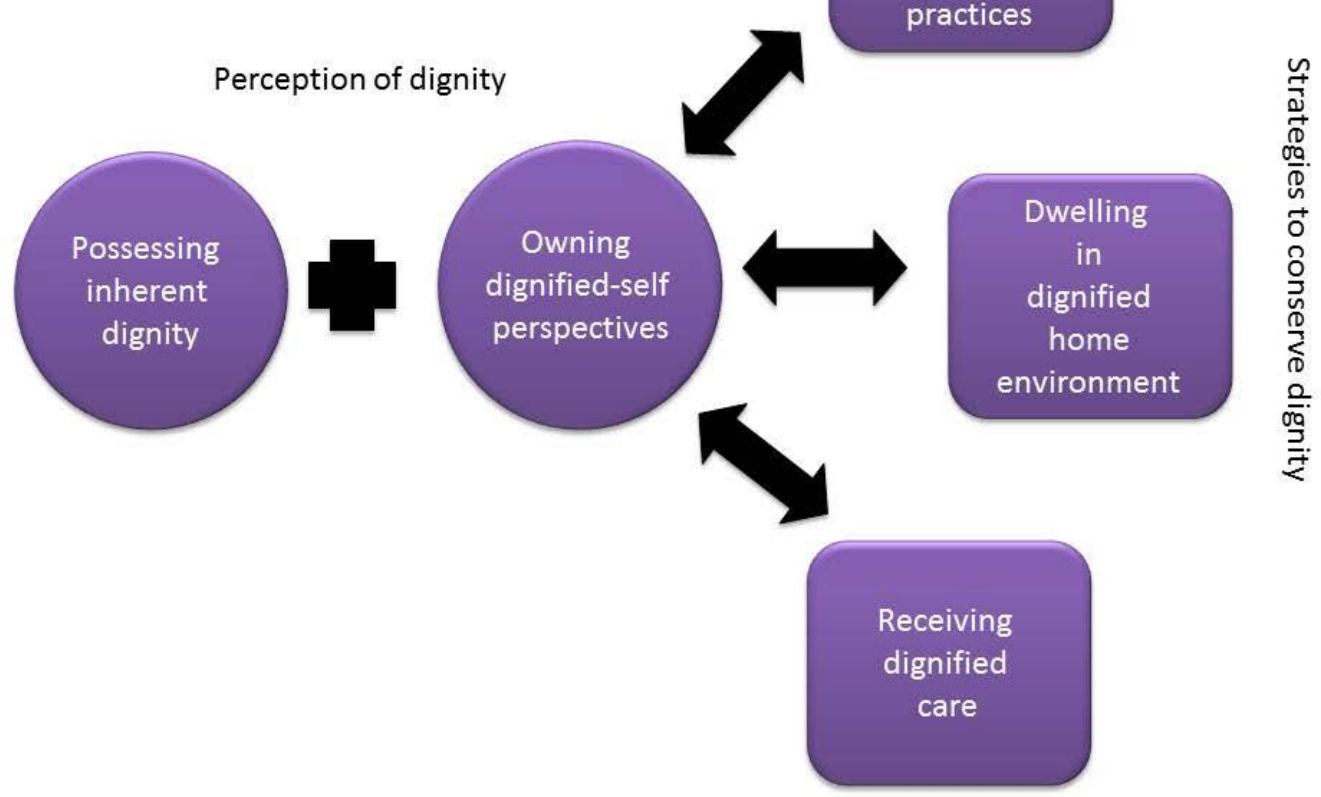

Figure 1. Framework of being dignified for elderly Chinese in a private nursing home 


\section{Perception of Dignity}

Although different researchers have categorised the concept of dignity differently, the intrinsic and extrinsic natures are embedded. This study revealed that inherent dignity is prominent but not as important to residents. The Western world views human dignity as a characteristic or property of individuals. In contrast, Chinese ethics, especially those rooted in Confucian teachings, typically construe dignity as worth that one acquires by behaving properly within relationships (Koehn \& Leung, 2008) [10]. Although the themes "possessing inherent dignity" and "owning dignified-self perspectives" seems to be related to the "self", what it deeply implies is relation with others. The participants verbally agreed that "As a human being, one has dignity since birth", which is partially consistent with the Western literature. In further interviews, it was found that the participant agreed that everyone is born with inherent dignity because he or she is a human being. 'One' should be dignified regardless of who one is or what one has. However, the 'one' applies to all human beings excluding the participant him or herself. To others, the mere existence of the physical body can indeed obtain the sense of inherent dignity and it cannot be lost. However, when inherent dignity applies to the 'self', it can be lost. In Confucian thought, the role of the 'self' is not to express and manifest itself (as in Western models), but to develop the internal moral self (Yu 1996) [32]. Possessing inherent dignity is an essence of being dignified for residents in nursing homes; however, they did not emphasize this very much. In order for one to develop this moral self, one needs to possess certain characteristics. These characteristics are identified as dignified-self perspectives in this study.

Participants agreed that the most important reason for them to choose a nursing home was because they did not want to be a burden to others - especially their family members. Although a nursing home was referred as " $a$ dumping place where one would idle till death” (Lee, 1997) [11] and the majority of the elderly in the community would not want to live in a nursing home, and their nursing home-related beliefs were found to be negative (Tse, 2007) [30], this study revealed that living in a nursing home is a way to make them dignified because it will lessen their sense of being burdens to others. Not being a burden to others is high on the list of Western cultural values (Callahan, 1997) [1], and this also applies to Chinese culture.

According to the classical views of Confucius and Mencius, longevity is not good in itself; rather, what is desirable in itself is a life of ren (benevolence), a life that will be remembered, respected, and honored in history. What matters is not life's quantity, but its quality, to be defined morally with reference to ren. Having unnecessary prolongation of life will decrease the quality of life and cause a burden to others. It causes the violation of ren. It is the matter of adding years to life or adding life to years. In certain ways, while the health professional can add years to life; by respecting the wills of the elderly - not having life-sustaining treatment - perhaps life can be added to those years.

\section{Strategies to Enhance Dignity}

To have dignity, one must feel worthy (Coventry 2006) [3]. Having perseverance in striving for one's goal by hard work is a highly valued virtue in traditional Chinese culture, especially in the philosophical system of Confucianism (Hwang 1977) [7]. In Chinese concepts, it is desirable to employ this strategy because only those who are able to endure hardship can obtain a sense of worthiness and thus dignity. Chinese culture is also characterised as collectivistic (Yu 1996; Lam 2015) [32, 33]. The residents' sense of dignity could be maintained through making some contribution to the nursing home. Thus, involving residents in participating in care (for the self and others) is recommended.

Dignified care is given by children and nursing staff. The participants valued the concept of xiao of their children. It means children expressing their love through frequent contacts, buying them something that the residents like. What is presented here is the concept of gongyang. Gongyang means reverent caring or providing sustenance in a respectful fashion (Knapp 2004) [9]. As family members play a significant role in generating dignity in residents, some strategies that could facilitate the visit of the family member(s) such as lengthening the visiting hours, arranging some inter-generational activities for the family members to participate in, or installing visual teleconferencing so that the residents can see their family members when they are unable to visit could be considered.

Residents commonly authorised and delegated the nursing staff to make decisions for them. Moreover, with their physical limitations, the residents perceived that they were relying on the nursing staff to perform many activities of daily life in the nursing home. With time, the nursing staff may develop a paternalistic attitude. When the staff is caring with such an attitude, the residents' sense of dignity will be intruded upon. The nursing staff should develop a mutually-respectful relationship instead of being paternalistic. In enhancing the particularistic dignity, the staff needs to explore the unique meaning of importance to the notion of living with dignity in the nursing home from the residents' perspectives.

A local Hong Kong study by Low et al. (2007) and Lam (2015) $[13,33]$ similarly indicated that privacy was perceived as not necessary at first, but further prompting identified that informants used their own efforts to support their privacy. This study revealed that physical privacy was perceived as necessary in maintaining the resident's dignity. Thus, increasing private areas such as constructing more single rooms or more partitions in private nursing homes are recommended.

As Shotton and Seedhouse (1998) [24] stated, the elderly may gain control by adjusting the environment to 
an older person's capability. By modifying the physical environment, such as installing more hand-rails or an elevator, they do not need to ask the nursing staff to assist them for every single event, their sense of being physically independent and not being a burden may increase, and thus dignity increases. This finding is very locally special as many private nursing homes in Hong Kong are operated in traditional village houses. These houses usually do not have an elevator. For those who are unable to use the stairs, the nursing staff need to lift them up or carry them down. They felt humiliated and indignant.

\section{Conclusions}

This study provided a better understanding of the lived experience of being dignified for elderly Chinese residents in a private nursing home in Hong Kong. A framework was developed to guide dignified practice in nursing homes. Moreover, some strategies for clinical practice were suggested. It is obvious that this study did not support Macklin's (2003) [14] finding which said that dignity is a useless concept which means no more than respect for persons or their autonomy. Dignity - for elderly Chinese people, at least - consists of a much wider horizon than that.

\section{Acknowledgements}

I would like to thank all the participants of this study, staff of the nursing home and Professor Esther Mok for their ceaseless support.

\section{REFERENCES}

[1] Callahan, D. (1997) The value of achieving a peaceful death. In C. K. Cassel , R. M. Leipzig, H. J. Cohen, E. B. Larson, D. E. Meier \& C. F. Capello (Eds.), Geriatric Medicine: an evidence-based approach $\left(4^{\text {th }}\right.$ ed.) (pp.351-358). New York: Springer.

[2] Chinn, P.L., \& Kramer, M.K. (1999) Theory and Nursing Integrated Knowledge Development $\left(5^{\text {th }}\right.$ ed.). St Louis: Mosby.

[3] Coventry, M. (2006). Care with dignity: a concept analysis. Journal of Gerontological Nursing, 32(5), 42-48.

[4] Fenton, E., \& Mitchell, T. (2002) Growing old with dignity: a concept analysis. Nursing Older People, 14(4), 19-21.

[5] Griffin-Heslin, V.L. (2005) An analysis of the concept dignity. Accident and Emergency Nursing, 13, 251-257.

[6] Haddock, J. (1996) Towards further clarification of the concept 'dignity'. Journal of Advanced Nursing, 24, 924-931.
[7] Hwang, K.K. (1977) The patterns of coping strategies in a Chinese society. Acta Psychologica Taiwanica, 19, 61-73.

[8] Jacelon, C.S., Connelly, T.W., Brown, R., Proulx, K., \& Vo, T. (2004) A concept analysis of dignity for older adults. Journal of advanced Nursing, 48(1), 76-83.

[9] Knapp, K.N. (2004) Reverent caring: the parent -son relationship in early medieval tales of filial offspring. In A.K. Chan, \& S.H. Tan (Eds.), Filial piety in Chinese thought and history (pp.44-70). New York: RoutledgeCurzon.

[10] Koehn, D., \& Leung, A. (2008) Dignity in Western versus in Chinese cultures: theoretical overview and practical illustrations. Business and Society Review, 113(4), 477-504.

[11] Lee, D.T.F. (1997) Residential care placement: perceptions among elderly Chinese people in Hong Kong. Journal of Advanced Nursing, 26, 602-607.

[12] Lincoln, Y., \& Guba, E. (1985) Naturalistic Inquiry. California: Sage.

[13] Low, L.P.L., Lee, D.T.F., \& Chan, A.W.Y. (2007) An exploratory study of Chinese older people's perceptions of privacy in residential care homes. Journal of Advanced Nursing, 57(6), 605-613.

[14] Macklin, R. (2003) Dignity is a useless concept. British Medical Journal, 327, 1419-1420.

[15] Mairis, E.D. (1994) Concept clarification in professional practice --- dignity. Journal of Advanced Nursing, 19, 947-953.

[16] Matiti, M.R., \& Trorey, G.M. (2004) Perceptual adjustment levels: patients' perception of their dignity in the hospital setting. International Journal of Nursing Studies, 41, 731-744.

[17] Nordenfelt, L. (2004) Dignity of the elderly: An introduction. Medicine, Health Care and Philosophy, 6, 99-101.

[18] Nordenfelt, L., \& Edgar, A. (2005) The four notions of dignity. Quality in Aging. Policy, Practice and Research, 6 (1), $17-21$.

[19] Peyrovi, H., Yadavar-Nikravesh, M., Oskouie, S.F., \& Bertero, C. (2005) Iranian student nurses' experiences of clinical placement. International Nursing Review, 52, 134-141.

[20] Polit, D.F. \& Beck, C.T. (2006) Essentials of Nursing Research: methods, appraisals, and utilization ( $6^{\text {th }}$ ed.). Philadelphia: Lippincott Williams \& Wilkins.

[21] Pullman, D. (1999) The ethics of autonomy and dignity in long-term care. Canadian Journal on Aging, 18 (1), 26-46.

[22] Pullman, D. (2001) Universalism, particularism and the ethics of dignity. Christian Bioethics, 7(3), 333-358.

[23] Richard, L., \& Morse, J.M. (2007) Read Me First for a user's guide to qualitative methods ( $2^{\text {nd }}$ ed.). Thousand Oaks: Sage Publications.

[24] Shotton, L., \& Seedhouse, D. (1998) Practical dignity in caring. Nursing Ethics, 5 (3), 246-255.

[25] Social Welfare Department. (2018). Services for the Elderly. Retrieved from http://www.swd.gov.hk/en/index/site_pubsvc/page_elderly/ 
sub_introducti/

[26] Speziale, H.J., \& Carpenter, D.R. (2007) Qualitative research in nursing: advancing the humanistic imperative. Philadelphia: Lippincott Williams \& Wilkins.

[27] Sulmasy, P. (2007) Human dignity and human worth. In J. Malpas \& N. Lickiss (Eds.). Perspectives in Human Dignity: A Conversation (pp.9-18). The Netherlands: Springer.

[28] Tadd, W., Bayer T., \& Dieppe, P. (2002) Dignity in health care: reality or rhetoric. Review of Clinical Gerontology, 12, $1-4$

[29] Teon, A. (2016). Filial Piety in Chinese Culture. The Greater China Journal. Retrieved from https://china-journal.org/2016/03/14/filial-piety-in-chineseculture/
[30] Tse, M.M.Y. (2007) Nursing home placement: perspectives of community-dwelling older persons. Journal of Clinical Nursing, 16, 911-917.

[31] van Manen, M. (1990) Researching lived experience: human science for an action sensitive pedagogy. Ontario: State University of New York Press.

[32] Yu, A.B. (1996) Ultimate life concerns, self, and Chinese achievement motivation. In M.H. Bond (Ed.), The Handbook of Chinese Psychology (pp.227-246). Hong Kong: Oxford University Press

[33] Lam, C. (2015). Development and validation of a quality of life instrument for older Chinese people in residential care homes (Doctoral dissertation, The Chinese University of Hong Kong (Hong Kong)). 